Check for updates

Cite this: RSC Adv., 2019, 9, 2353

\title{
Electrical control of liquid metal amoeba with directional extension formation $\uparrow$
}

\author{
Liang Hu, (D) ${ }^{* a}$ Xi Zhao, ${ }^{b}$ Jiarui Guo ${ }^{a}$ and Jing Liu*bc
}

In this study, an electric field was used to regulate and control pseudopodia-like extensions of a liquid metal-Al (LM-Al) droplet in certain directions. The results suggest that in certain electric fields, the LM-Al droplets tend to generate extensions perpendicular to the electric field; the underlying mechanism arises from the specific surface tension imbalance induced by the electric field. The influence of varying electric field intensity and Al content on the LM-Al transformations was also evaluated; the LM-Al droplets displayed specific and distinct behaviors according to each experimental configuration; this further proved the feasibility of using electric fields for controlling LM-Al transformations. The entire study provides a promising and practical method for control of LM amoeba-like transformations, which are valuable for further development of soft robots and devices.

Received 6th December 2018 Accepted 26th December 2018

DOI: $10.1039 / c 8 r a 10044 f$

rsc.li/rsc-advances soft devices functioning as actuators or electrical connectors. However, the extension direction of the LM amoeba is mainly dependent on the Al distribution, which is not yet easy to control. In practical applications, directional controllability is a desirable property for most actuators or robots. For LM amoeba, the pseudopodia-like extensions may work as mechanical hands to perform some specific tasks such as electrical connection or actuation, which also requires directional controllability. In this study, we intend to utilize an electric field to regulate the LM extension to make it more direction-controllable. The detailed experiments and results have been discussed in the following sections.

\section{Results and discussion}

In this study, LMs and LM-Al droplets were prepared as described in our previous report. ${ }^{5}$ Briefly, LM-Al droplets were prepared by dissolving a small piece of Al foil $(1.6 \mathrm{mg})$ in a $500 \mathrm{~mL} \mathrm{Ga}{ }_{65} \mathrm{In}_{22} \mathrm{Sn}_{13}$ droplet in $0.5 \mathrm{~mol} \mathrm{~L}^{-1} \mathrm{NaOH}$. The experimental phenomenon was determined by a digital video equipment, (Sony HDR-PJ670). An approximately uniform electric field was provided by two electrodes, which were connected to a $10 \mathrm{~V}$ power supply and fixed at a distance of $17.5 \mathrm{~cm}$. A graphite plate $(10 \mathrm{~cm} \times 10 \mathrm{~cm} \times 1 \mathrm{~cm})$ was placed right in the middle of the two electrodes as illustrated in Fig. 1A. When the graphite plate was placed in the electric field, bubbles were generated at both edges of the graphite plate. In details, there were more bubbles on the left edge than those on the right (ESI Fig. S1, $\dagger$ upper). The voltage between the electric electrodes and the adjacent graphite edges has been measured at around $4.95 \mathrm{~V}$, which is large enough to induce water hydrolysis $(1.229 \mathrm{~V}$ required). Thus, this suggests that the electric electrodes should form new electrode pairs with the adjacent graphite edges for 
water electrolysis. When the LM-Al droplet was placed on graphite and kept transforming in the electric field, abundant bubbles were observed on the left edge of the graphite plate close to the positive pole. However, on the right edge, few bubbles were observed (ESI Fig. S1, $\dagger$ lower). It is believed that the graphite plate was cathodically polarized by the $\mathrm{Al}$ reaction $\left(\mathrm{Al}-3 \mathrm{e}=\mathrm{Al}^{3+}\right)$ through contact between the LM-Al droplet and the graphite. Thus, the hydrogen evolution reaction was enhanced and oxygen evolution was inhibited.

After the Al foil was fully dispersed in the droplet without visible large debris, the LM-Al droplet was placed on graphite. In the present study, two types of LM-Al behaviors were observed and statistically categorized based on their appearance in the electrical field. In Type 1, the LM-Al droplet was placed close to the positive pole (at a distance of around $5 \mathrm{~cm}$, Fig. 1C). At first, it deformed due to the imbalance in surface tension induced by the integrated effects from the interaction of the $\mathrm{Al}$ and the graphite plate (ESI S1 $\dagger) .{ }^{5}$ After several seconds, the left side facing the positive pole suddenly became metallic and bright. Then, the droplet quickly ran to the positive pole (Fig. 1B). Since the electric field should be generally uniform, the electric field intensity should not result in these different behaviors. Thus, it was inferred that the droplet was partially reduced by abundant anions close to the positive pole. As a result, the droplet should be negatively charged in $\mathrm{NaOH}$ and should be attracted to the positive pole. The driving

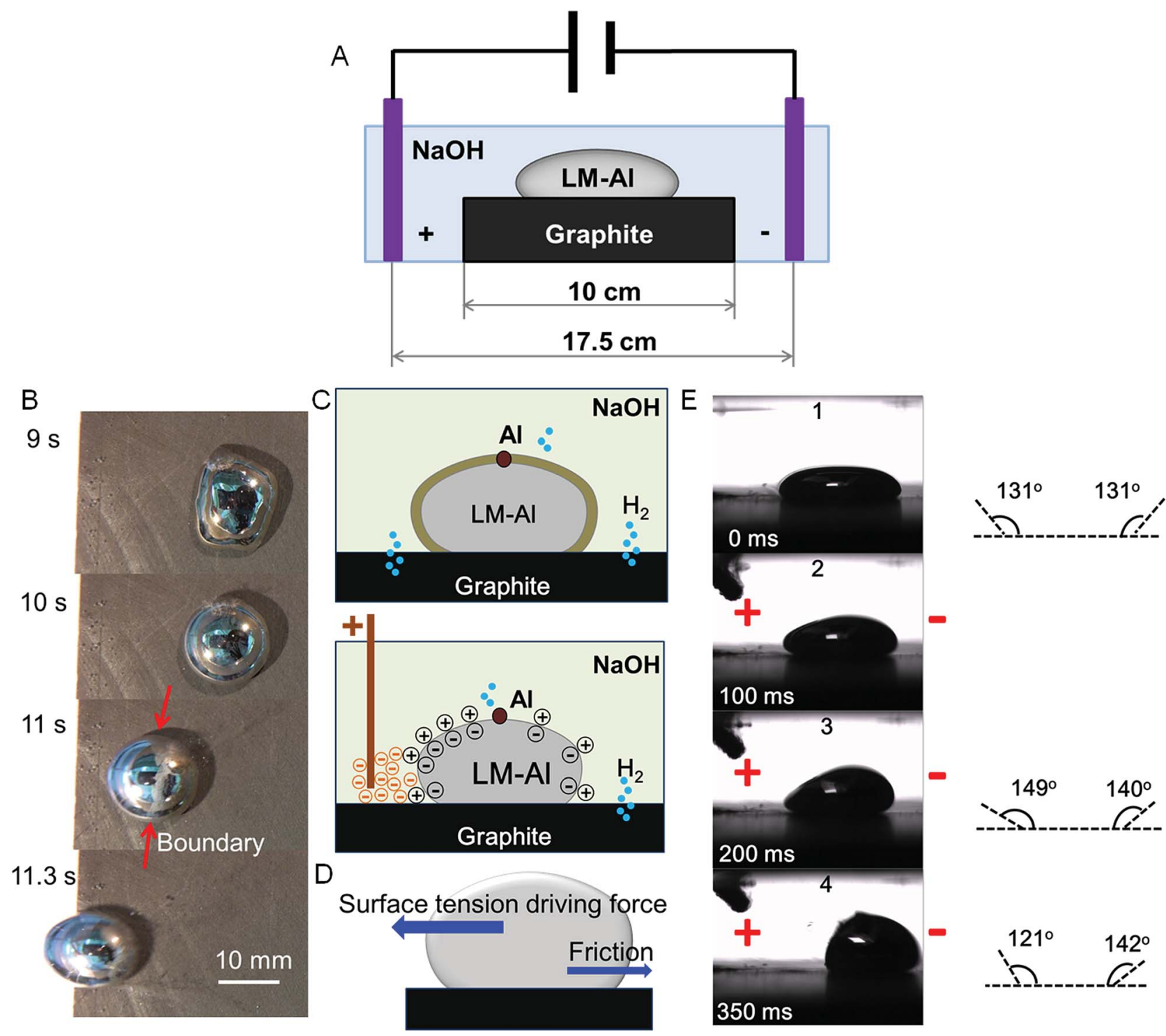

Fig. 1 (A) A schematic of the experimental setup. (B) The LM-Al droplet close to the positive pole became reduced and ran to the positive pole. The red arrows indicate the boundary where half of the droplet was reduced and the other half was covered with oxide. The scale bar is $1.5 \mathrm{~cm}$. (C) A schematic illustration of how the LM-Al was reduced by the positive pole. (D) A force analysis of LM-Al in Type 1 behavior. (E) A side-view of the LM droplet when the positive pole was moved close. Left, consecutive snapshots of a liquid metal droplet on graphite with electric field induced deformation. The positive pole is marked red. Right, the contact angles of the LM droplet with graphite measured in images 1 , 3, and 4 (left) respectively. 


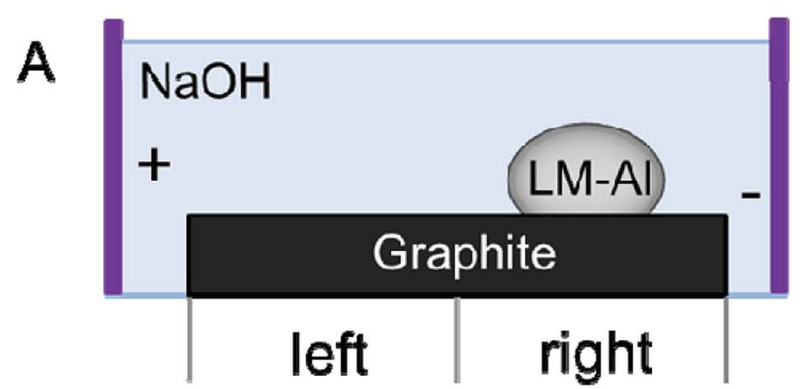

\section{left}
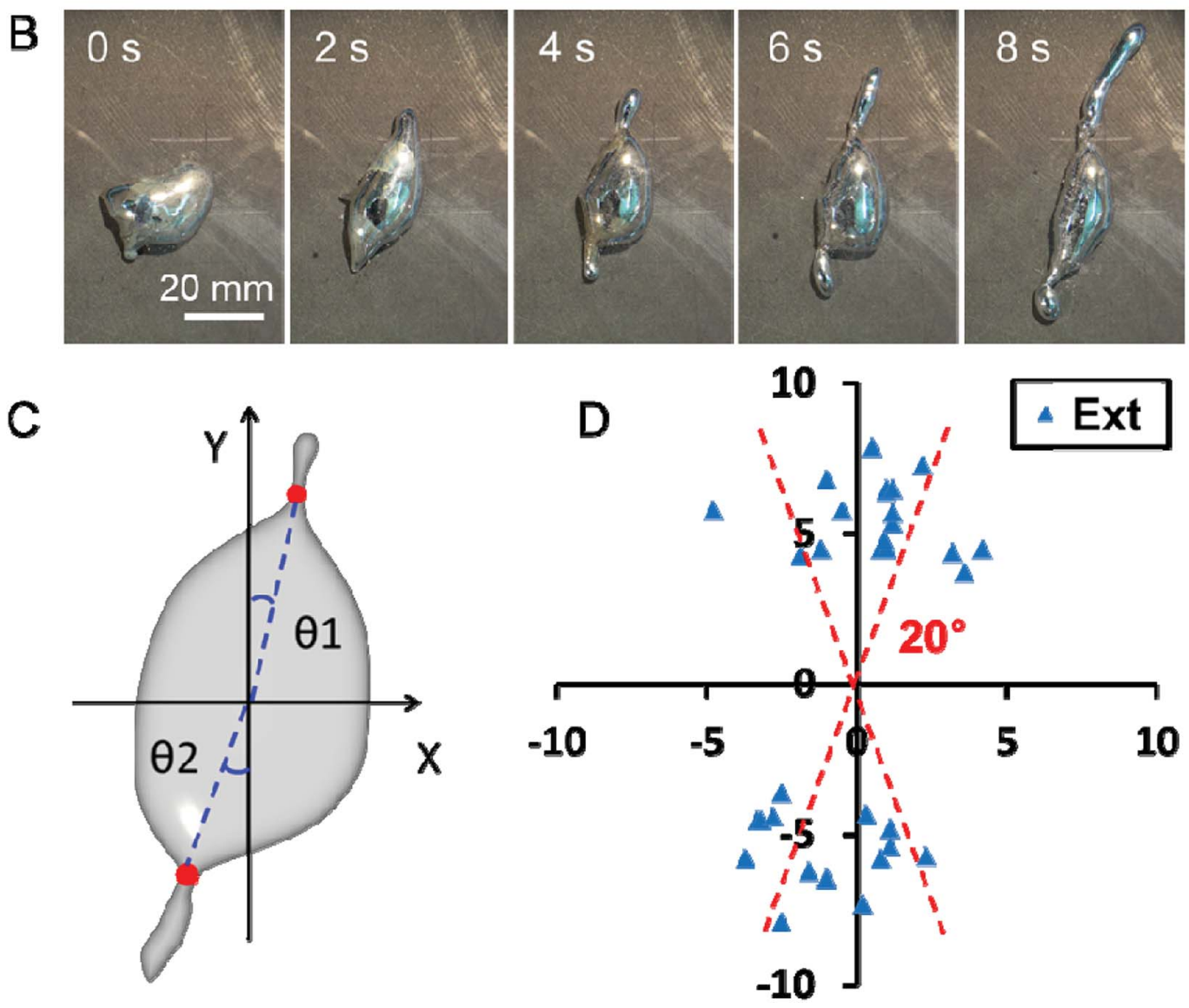

Fig. 2 (A) A schematic of the experimental setup in Type 2 behavior. (B) A snapshot of a LM-droplet with two perpendicular extensions. The scale bar is $2 \mathrm{~cm}$. (C) An illustration of the calculation method for the extension directions. (D) The statistics of extension direction for LM-Al droplets in the electric field. The blue triangle (Ext) represents the location of extension formation according to the calculation method in (C).

force is provided by the surface tension gradient, which is derived from the electric field-induced surface charge imbalance. This process has been investigated and analyzed in many previous studies. ${ }^{1,6}$ Thus, the detailed analysis has not been discussed here. A general force analysis of the reduced LM-Al in the electric field is shown in Fig. 1D. To confirm this assumption, the contact angles of the LM droplet were investigated (Fig. 1E). When the positive pole was moved close to the left side of the droplet, the contact angle also decreased at the left side (Fig. 1E). The droplet appeared to quickly contract from a flat droplet into a more spherical droplet; this indicated an increase in surface tension. Based on the contact angle measurement, together with the metallic droplet surface, it seems that the droplet is reduced, and the surface tension is regained at the left side. This is also consistent with our observation that the droplet is attracted to the positive pole.

In Type 2, when the LM-Al droplet was placed on the right half of the graphite surface close to negative pole (at a distance less than $9 \mathrm{~cm}$, Fig. 2A), it seemed that the pseudopodia-like extensions could be formed in specific directions. For all the droplets which successfully gave out extensions, the vast majority (15 in 17) formed two extensions (Fig. 2B). The time required for the initiation of both extensions was around $1.2 \pm$ $0.3 \mathrm{~s}(n=8)$. To quantitatively describe the extension direction, a calculation method was defined, as illustrated in Fig. 2C. Initially, the center of the main droplet (the intersection of the two midlines of the droplet in the $X$ and $Y$ directions) was set as the origin point; following this, each extension initiation point 

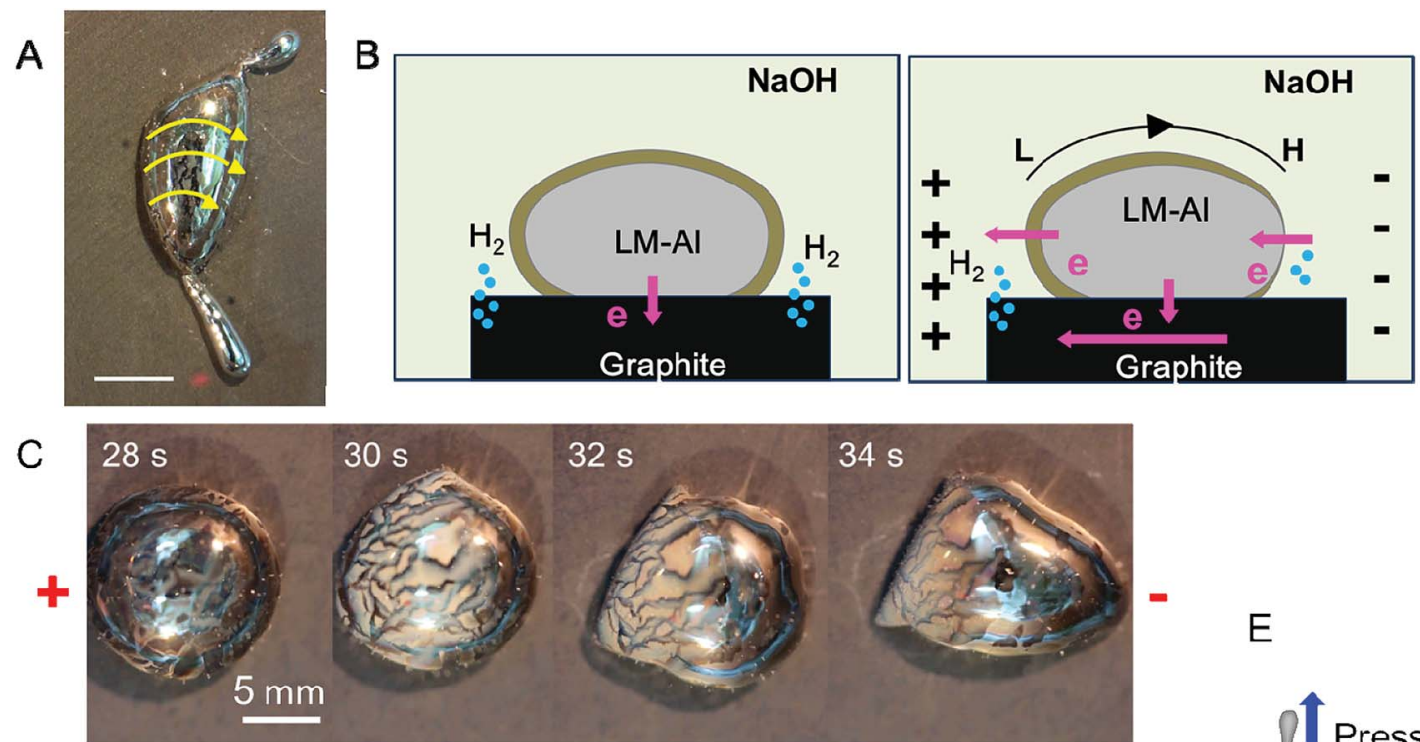

$\mathrm{D}$
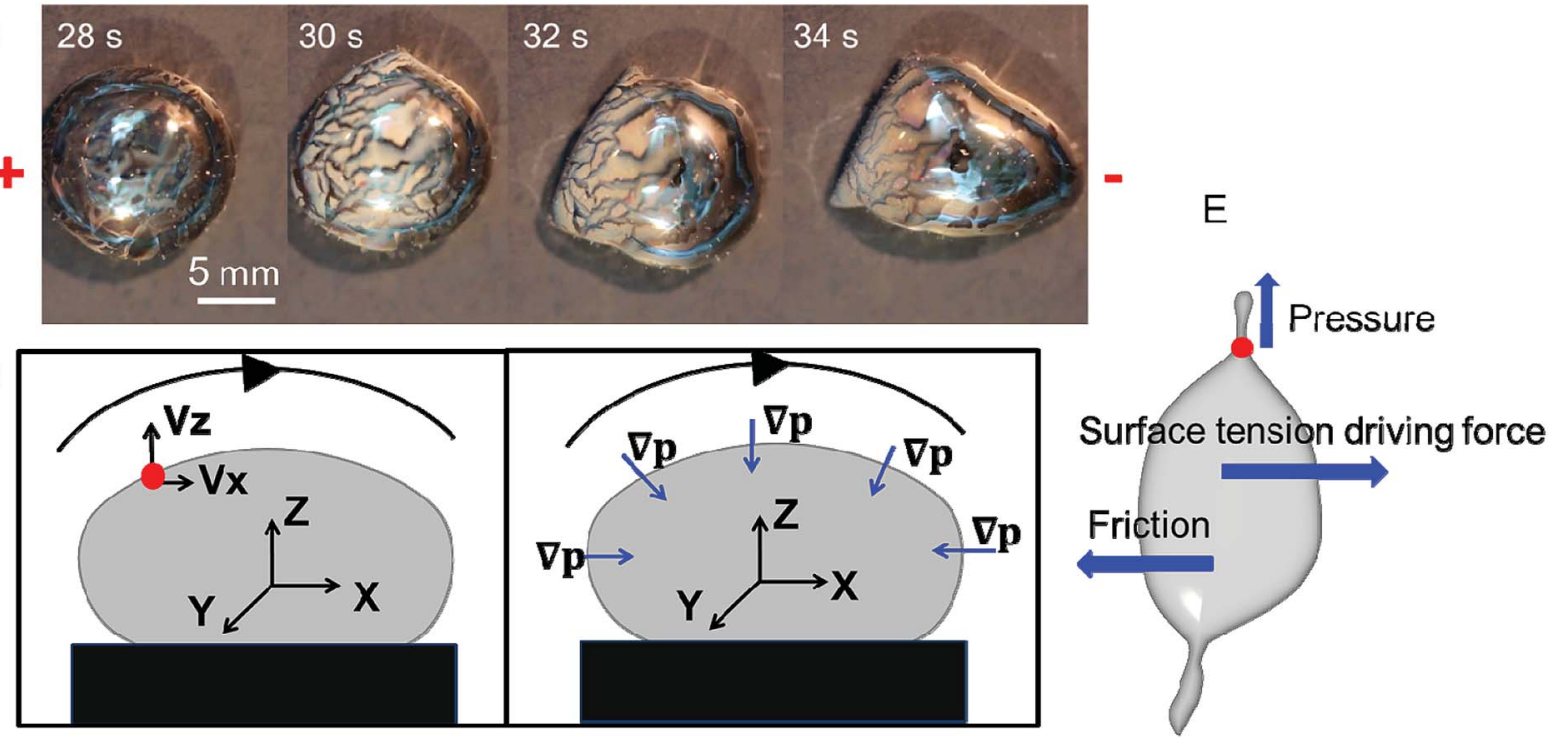

Fig. 3 (A) The surface rolling flow along the main body of the LM-Al droplet. (B) A schematic diagram of the surface tension gradient formed due to the electric field. The black arrow indicates the surface flow direction. ' $\mathrm{L}$ ' indicates low surface tension and ' $\mathrm{H}$ ' indicates high surface tension. Pink arrows indicate the electron flow. (C) The surface oxide distribution imbalance in the electric field. (D) A force analysis of the droplet during rolling surface flow. (E) A force analysis of the LM-Al droplet during surface flow from left to right.

(red dots) and the origin point were connected; the angle between this line and the $Y$ axis $(\theta)$ was measured and taken as the extension direction angle. In statistics, most extensions (22 in 33) were formed in a direction almost perpendicular $\left(<20^{\circ}\right)$ to the electric field (Fig. 2D). For comparison, when the electric field was powered off, the extension formation was also investigated. These results showed that most droplets put out three extensions rather than two. Only 4 of the 14 extensions were perpendicular $\left(<20^{\circ}\right)$ to the electric field; this was significantly lower than that with the electric field powered on. Moreover, no directional specificity was revealed in these extensions. These results imply that the extension forming direction should be related to the electric field direction.

The mechanism underlying this directional control related to the electric field is investigated. Based on our observations, during the extension of the perpendicular extensions, the surface of the LM-Al main droplet appeared to flow from left to right along the electric field lines (Fig. 3A, ESI Movie S1†); this suggested that the left part of the surface had lower surface tension due to the Marangoni effect. It is predicted that the different surface tensions at each end are due to an imbalance in surface oxide distribution. Before the electric field is applied, the LM-Al and the graphite should form a corrosion cell. The LM droplet works as an anode and loses electrons. The graphite works as a cathode and receives electrons (Fig. 3B, left). When the electric field is applied, although the electron flow from the LM-Al to the graphite should still exist, the LM-Al should also be polarized. Similarly, as abovementioned, graphite in the electric field should also form electrode pairs with adjacent electrical poles; thus, the left side of the droplet loses electrons and becomes further oxidized; the right side receives electrons and becomes somewhat reduced. In this way, the surface tension on the left should decrease due to more surface oxide formation, whereas on the right side, it may increase (Fig. 3B, right). As a result, the left surface would flow to the right due to a surface tension imbalance. In the experimental study, when the electric field was powered on, the left part of the droplet, facing the positive pole, was suddenly covered with obvious surface oxide, whereas the right part was not obviously changed (Fig. 3C). This observation provides proof for our assumption. Thus, it is confirmed that it is the electric field that induces the surface flow along the electric field lines.

During surface flow, the droplet tended to give out two extensions along the perpendicular direction. This 


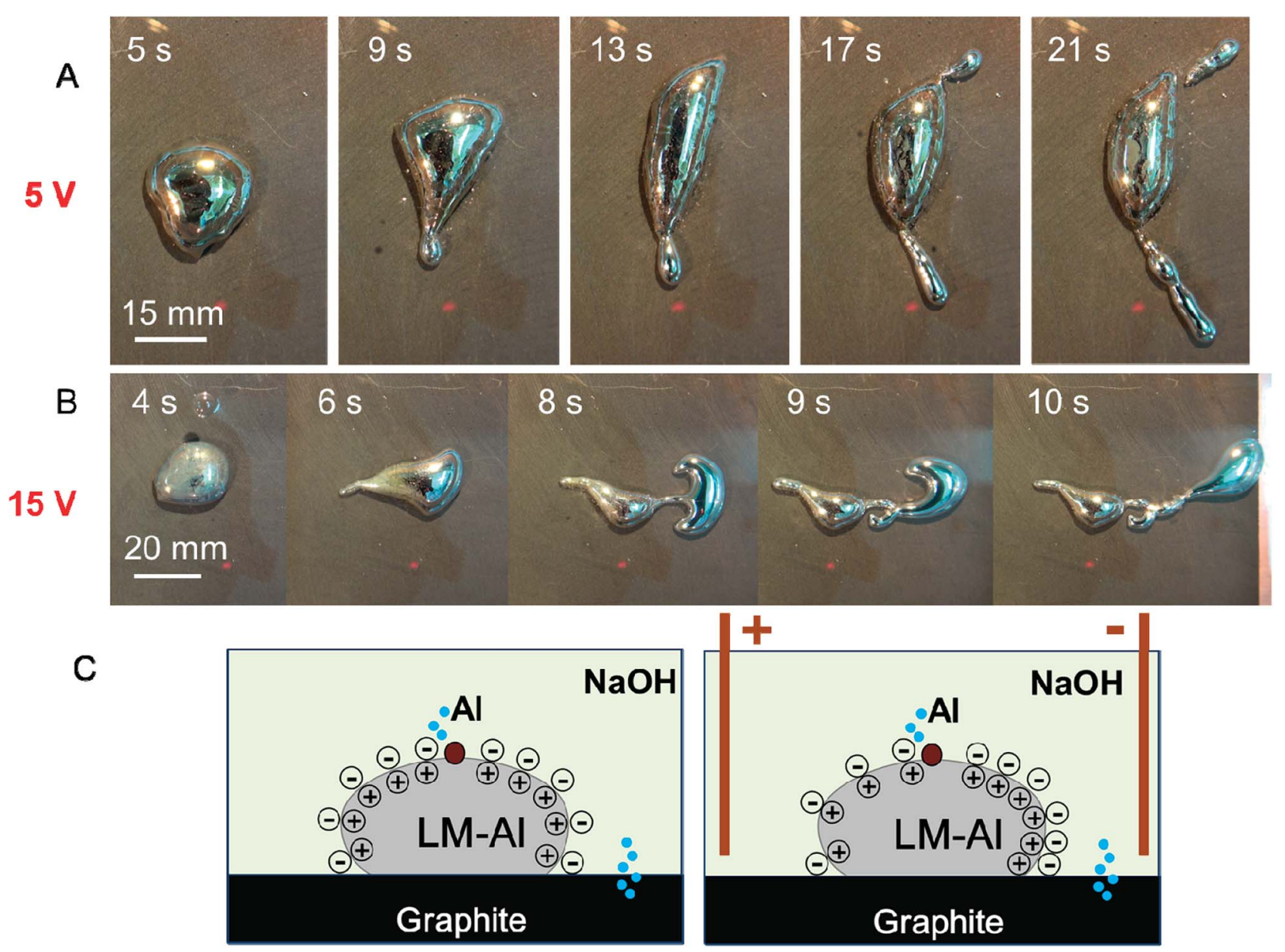

Fig. 4 Consecutive images of the typical LM-Al droplet $(1.6 \mathrm{mg} / 500 \mu \mathrm{L}$ ) transformations in an electric field powered by a $5 \mathrm{~V}$ (A) and a $15 \mathrm{~V}$ (B) power supply. (C) A schematic of the LM-Al droplet surface charge imbalance induced by the electric field.

phenomenon strongly implies that the extension direction may be related to the surface flow mode. To confirm this assumption, a force analysis was conducted. According to the NavierStokes equation, the momentum conservation of an incompressible fluid flow parcel is

$$
\rho \frac{\mathrm{d} V}{\mathrm{~d} t}=\rho g-\nabla p+\mu \nabla^{2} V
$$

where $\rho$ is the fluid density, $V$ is the velocity of the flow parcel, $p$ is the pressure, $g$ is the acceleration due to gravity, and $\mu$ is the kinematic viscosity. In the $X, Y, Z$ dimensions,

$$
\begin{gathered}
\frac{\mathrm{d} V}{\mathrm{~d} t}=\frac{\partial V}{\partial t}+u \frac{\partial V}{\partial x}+v \frac{\partial V}{\partial y}+w \frac{\partial V}{\partial z} \\
\nabla^{2} V=\frac{\partial^{2} V}{\partial x^{2}}+\frac{\partial^{2} V}{\partial y^{2}}+\frac{\partial^{2} V}{\partial z^{2}}
\end{gathered}
$$

where $u, v$ and $w$ are the velocity of fluid flow in the $X, Y$ and $Z$ directions, respectively. For the droplets in our study, the small parcels of LM have obvious surface motion in the $X$ and $Z$ directions, whereas they have no motion in the $Y$ direction
(Fig. 3D). Thus, $\frac{\partial V}{\partial y}=0, \frac{\partial^{2} V}{\partial y^{2}}=0$. The surface flow is considered steady at a certain time, $\frac{\partial V}{\partial t}=0$. In the $X$ and $Y$ directions, the force of gravity is not considered. So, $\rho g=0$. Thus, the equation can be deduced as follows:

In the $X$ and $Z$ directions,

$$
\nabla p_{x}=\mu \frac{\partial^{2} V}{\partial x^{2}}-\rho u \frac{\partial V}{\partial x}
$$

$$
\nabla p_{z}=\rho g+\mu \frac{\partial^{2} V}{\partial z^{2}}-\rho w \frac{\partial V}{\partial z}
$$

While in the $Y$ direction where there is no surface flow or motion,

$$
\nabla p_{y}=0
$$

It is concluded that external pressure only exists in the $X$ and $Z$ directions due to the surface flow motion. No external 


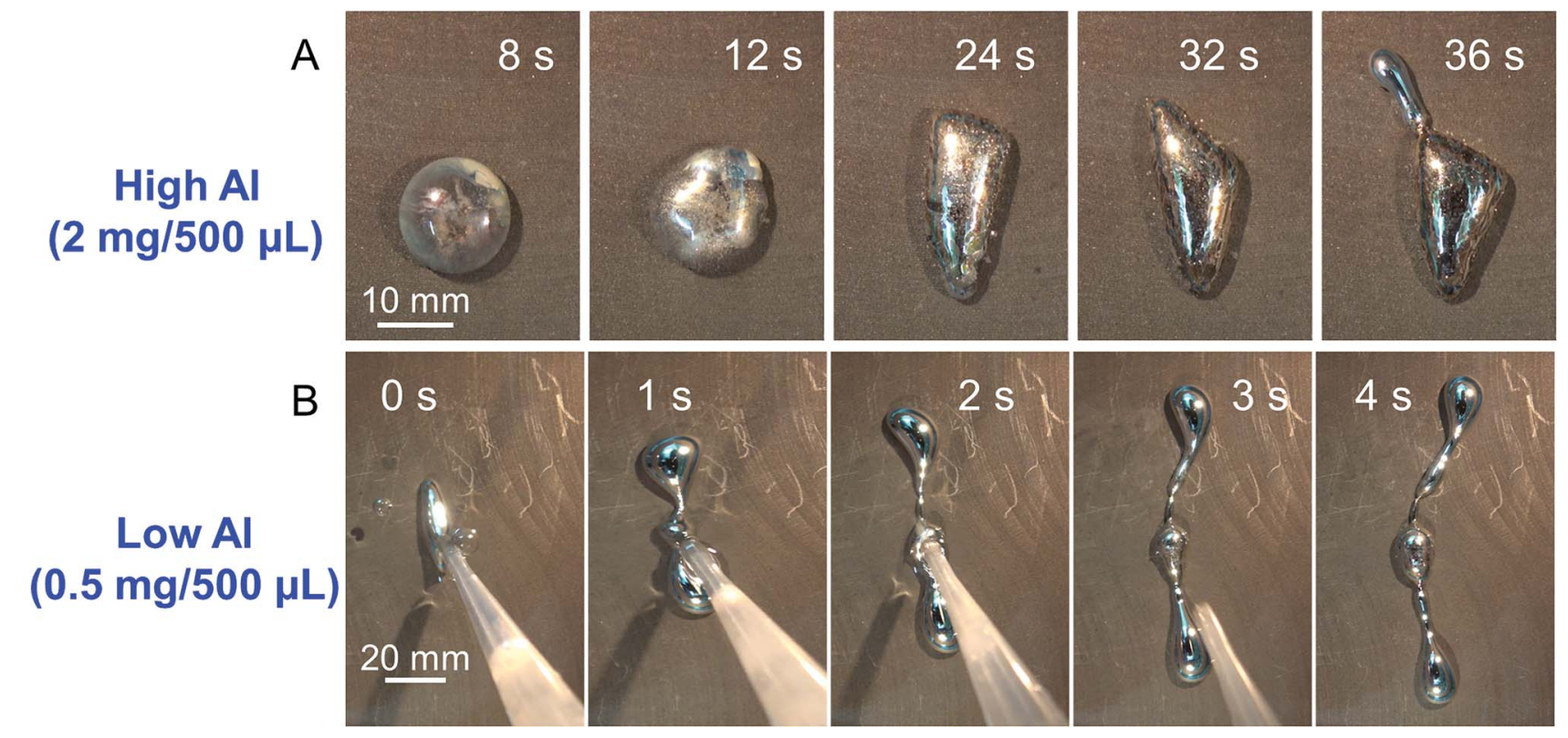

Fig. 5 The LM-Al droplet transformation with high $(2.0 \mathrm{mg} / 500 \mu \mathrm{L}, \mathrm{A})$ and low $(0.5 \mathrm{mg} / 500 \mu \mathrm{L}, \mathrm{B}) \mathrm{Al}$ content in an electric field powered by a $10 \mathrm{~V}$ power supply.

pressure exists in the $Y$ direction (Fig. 3D). As the surface tension gradually decreases, the droplet should spread out as discussed in previous studies. ${ }^{7}$ In the $X$ and $Z$ directions, the external pressure should prevent this spreading behavior. However, in the $Y$ direction, the droplet is more likely to spread at the places where external pressure is lower. Thus, the inner LM was propelled outward by the internal pressure at the extension forming corner (red dot). The whole droplet is subjected to these forces, including the surface tension driving force induced by the imbalance in surface tension as well as friction from the substrate and the surrounding liquid (Fig. 3E). Overall, the electrical field is proven to have significant effect in inducing these amoeba pseudopodia-like extensions in specific directions.

As the electric field may affect the surface oxide distribution and further the surface tension of LMs, we have examined the motion properties of various LM-Al droplets under different electric field intensities. For two electrodes at the same distance, two sets of voltages, $15 \mathrm{~V}$ and $5 \mathrm{~V}$, were used to evaluate the effect of the electric field intensity on the liquid droplet motion. Herein, we have also used a LM-Al droplet $(1.6 \mathrm{mg} / 500$ $\mu \mathrm{L})$ as an example. When the LM-Al droplet was placed on the left part of the plate, it also became half-reduced and ran to the positive pole ( 8 in 10). On the middle and right parts of the plate, 4 in 10 droplets also give out perpendicular extensions (Fig. 4A), which is a lower fraction than that at $10 \mathrm{~V}(22$ in 33). The times required for obvious two extension formation were generally longer $(3.3 \pm 1.2 \mathrm{~s}, n=4)$. This implies that the electric field is relatively weak, which may have a smaller effect on the surface flow for perpendicular extension formation. When the power was set at $15 \mathrm{~V}$, the LM-Al droplet also ran to the positive pole when placed on the left part of the plate. However, when the droplet was placed on the right part of the plate, its behavior became different. Although the droplet was still locally affected by Al oxidation and small perpendicular extensions formed, the main body of the droplet became elongated with a dragged-out tail and slid to the negative electric pole ( 8 in 10) (Fig. 4B); the dragged-out tail-like end was observed to be oxidized, similar to that shown in Fig. 3C, and did not move as fast as the right end of the LM droplet. Thus, the droplet appeared elongated. This motion was similar to that of the LM droplet in the electric field, in which the motion of the left part was hindered by the surface oxide to some degree (ESI Fig. S2 $\uparrow$ ), whereas the right part of the droplet quickly ran to the negative pole. ${ }^{7}$ The driving force of the whole droplet to the negative pole is likely the electric fieldinduced surface tension force (Fig. 4C). Briefly, the electric field-induced surface charge imbalance can induce a surface tension gradient along the droplet by changing the potential difference $(V)$ across the electric double layer $\left(\gamma=\gamma_{0}-\frac{1}{2} c V^{2}\right)$, which propels the droplet to the right based on the YoungLaplace equation $(P=2 \gamma / r) .^{6}$ Thus, the droplet was driven to the negative pole. Even in the $5 \mathrm{~V}$ electric field, the droplets also tended to move to the negative pole, but at a much lower speed (around $0.3 \mathrm{~cm} \mathrm{~s}^{-1}$ ). On the other hand, in the $15 \mathrm{~V}$-induced electric field, the droplets moved at a speed of around $0.8 \mathrm{~cm}$ $\mathrm{s}^{-1}$; this implied that they were more significantly affected by the electric field. These results suggest that the electric field not only plays the key role in inducing the perpendicular extensions but also provides the driving force for the whole droplet motion.

In this study, we also evaluated the impact of $\mathrm{Al}$ content in LM-Al droplets undergoing this direction-controllable transformation. Herein, we take the droplets at $10 \mathrm{~V}$ as an example. On the left part of the graphite plate, the behaviors of LM-Al droplets with different Al content were consistent. They all ran to the positive pole after being reduced. When the Al content was higher $(2 \mathrm{mg} \mathrm{Al} / 500 \mu \mathrm{L} \quad \mathrm{LM})$, no obvious 
pseudopodia formed during the first 10 seconds after the droplet was placed on the graphite although it kept deforming (10 in 10). As Al was continuously consumed, the droplets gradually put out extensions. This generally took around $21.2 \pm$ $8.6 \mathrm{~s}(n=5)$ (Fig. 4C). Most of the extensions (9 in 17) were also perpendicular. It has been discussed that LM-Al droplets with a higher Al content usually have high surface tension, which makes it more difficult to put out extensions due to the high surface pressure according to the Young-Laplace's equation. ${ }^{5}$ In this study, LM-Al droplets with higher surface tension should also be affected. Thus, it took longer to put out extensions. When the $\mathrm{Al}$ content was even higher ( $3 \mathrm{mg} \mathrm{Al} / 500 \mu \mathrm{L} \mathrm{LM})$, the droplets became more spherical and ran to the positive pole; this was similar to the case without an electric field. ${ }^{5}$ When the Al content was lower $(0.5 \mathrm{mg} \mathrm{Al} / 500 \mu \mathrm{L} \mathrm{LM})$, all the droplets ( 5 in 5) quickly extended before being completely placed on graphite (Fig. 5). This is likely due to quick reduction of the surface tension by interaction with the graphite plate. The droplets also tended to extend in perpendicular directions (4 in 5). It seems that the extension direction is more likely to be perpendicular when the $\mathrm{Al}$ content is lower. A possible reason is the weaker $\mathrm{Al}$ reaction, which has less influence on altering the surface tension as compared to the electric field-induced driving force.

\section{Conclusion}

In this study, an electric field was applied to investigate directional control of an LM amoeba-like transformation. The results suggest that the transformation and pseudopodia-like extension direction of LM-Al droplets can be regulated, to some degree, by the electric field and the Al content. Specifically, by regulating the electric field intensity, the extension of the droplet can be formed in a direction approximately perpendicular to the electric field; this is promising for control of liquid metal-based soft machines or robots. In addition, physical and chemical analysis of the whole system provides additional fundamental knowledge of LM amoeba transformations that is valuable for future soft robot design.

\section{Methods and materials}

\section{LM-Al droplet transformation on graphite}

All the liquid metal (LM) droplets used in these experiments were GaInSn alloy prepared from gallium, indium and tin with purities of $99.99 \%$. These raw materials, with mass ratios of $67: 12: 13$, respectively, were added to a beaker and then heated to $100{ }^{\circ} \mathrm{C}$. A magnetic stirrer was used to stir the mixture uniformly after the metals were all melted. All the $\mathrm{NaOH}$ electrolyte used in this study was freshly made at $0.5 \mathrm{mM}$. The graphite plate had a purity of $99.9 \%$ and was $10 \mathrm{~cm} \times 10 \mathrm{~cm} \times$ $1 \mathrm{~cm}$ in length, width and height, respectively.

As the aluminum foil was too light to weigh, in our experiments, a large piece of aluminum foil was weighed and then cut into small pieces of the same size $(1.6 \mathrm{mg}=1$ piece). A drop of LM was initially injected into an $\mathrm{NaOH}$ solution in a glass Petri dish. A small piece of $\mathrm{Al}$ foil was then placed in contact with the LM droplet using forceps. Thus, Al was attached to the LM droplet and gradually broken into smaller granules as gallium would destroy the inter-granular bonds of the $\mathrm{Al}$ foil and penetrate into the $\mathrm{Al}$ grain boundaries. ${ }^{8}$ Some of the $\mathrm{Al}$ granules were dispersed inside the LM, whereas others accumulated together into big granules on the droplet surface. When the $\mathrm{Al}$ foil was dispersed into small granules without apparent large debris, the LM droplets containing $\mathrm{Al}$ (LM-Al droplets for short) were gently transferred by sucker onto the graphite substrate immersed in a $\mathrm{NaOH}$ solution. Following this, the transformations of the LM-Al droplets were observed and determined by digital video equipment (Sony HDR-PJ670). In the experiments, unless otherwise indicated, it was ensured that the graphite surface was horizontal to avoid interference from gravity.

\section{Conflicts of interest}

The authors declare that they have no competing interests that might be perceived to influence the results and/or discussion reported in this article.

\section{Acknowledgements}

This work was supported by the 111 Project (Project No.: B13003) and the National Natural Science Foundation of China (Grant No. 81801794).

\section{References}

1 L. Sheng, J. Zhang and J. Liu, Adv. Mater., 2014, 26(34), 6036. 2 J. Zhang, Y. Yao, L. Sheng and J. Liu, Adv. Mater., 2015, 27(16), 2648; S.-Y. Tang, V. Sivan, K. Khoshmanesh, A. P. O'Mullane, X. Tang, B. Gol, N. Eshtiaghi, F. Lieder, P. Petersen and A. Mitchell, Nanoscale, 2013, 5(13), 5949.

3 S. A. Chechetka, Y. Yu, X. Zhen, M. Pramanik, K. Pu and E. Miyako, Nat. Commun., 2017, 8, 15432.

4 D. Kim and J. B. Lee, J. Korean Phys. Soc., 2015, 66(2), 282.

5 L. Hu, B. Yuan and J. Liu, Sci. Rep., 2017, 7(1), 7256.

6 S.-Y. Tang, K. Khoshmanesh, V. Sivan, P. Petersen, A. P. O'Mullane, D. Abbott, A. Mitchell and K. Kalantarzadeh, Proc. Natl. Acad. Sci. U. S. A., 2014, 111(9), 3304.

7 L. Hu, L. Wang, Y. Ding, S. Zhan and J. Liu, Adv. Mater., 2016, 28(41), 9210.

8 D. O. Flamini, S. B. Saidman and J. B. Bessone, Corros. Sci., 2006, 48(6), 1413; A. V. Ilyukhina, O. V. Kravchenko, B. M. Bulychev and E. I. Shkolnikov, Int. J. Hydrogen Energy, 2010, 35(5), 1905. 